DOI: https://doi.org/10.46380/rias.v3i2.94

Vol. 3 No. 2, julio-diciembre 2020, pp. 98-109

\title{
Revitalización del uso de plantas alimenticias en la chakra kichwa Centro de Turismo Comunitario Sinchi Warmi.*
}

Revitalization of the use of food plants in the chakra kichwa of Sinchi Warmi Community

Tourist Centre.

Revitalização do uso das plantas alimentícias do kichwa chakra: Centro de Turismo Comunitário Sinchi Warmi.

María José Estupiñán Hernández / Universidad UTE, Ecuador/ mariajose.estupinan97@gmail.com

Recibido: 11/9/2020Ａceptado: 26/10/2020 Publicado: 30/12/2020

\section{RESUMEN}

La revitalización cultural ha permitido que los conocimientos, prácticas y saberes ancestrales, en riesgo de desaparecer, sean revalorizados y transmitidos a futuras generaciones para el manejo sustentable de sus recursos. En este contexto, la investigación tuvo como objetivo diseñar una propuesta para la revitalización del uso de plantas alimenticias en la chakra kichwa del Centro de Turismo Comunitario Sinchi Warmi, localizado en la parroquia Puerto Misahuallí, provincia Napo. Las metodologías implementadas para el levantamiento de la información fueron la etnografía y la etnobotánica, las cuales permitieron indagar sobre la interacción social histórica de esa comunidad dentro del sistema tradicional productivo agrícola chakra a partir del diálogo de saberes, con la finalidad de consolidar conocimientos científicos y tradicionales, y desarrollar acciones enfocadas al manejo y cultivo de plantas; teniendo en cuenta al patrimonio cultural inmaterial presente. Como resultados, se obtuvo el inventario de 60 especies vegetales con propiedades alimenticias y medicinales; y se elaboró con este una guía de plantas alimenticias y una aplicación móvil para el uso de ellas en la comunidad o por turistas y visitantes. Lo anterior ratifica a la revitalización cultural como una alternativa efectiva para la salvaguarda de los saberes ancestrales de estas comunidades.

Palabras clave: etnobotánica, etnografía, interpretación ambiental, patrimonio cultural y natural, saberes ancestrales

\footnotetext{
* Segundo lugar del I Talller Nacional Estudiantil sobre Medio Ambiente - Ecuador 2020
} 


\section{ABSTRACT}

The cultural revitalization has allowed that knowledge, practices and ancestral knowledge that are at risk of disappearing, can be revalued and passed to future generations for the sustainable management of their resources, in this context, this investigation had the objective of designing a proposal of revitalization of the use of edible plants in the kichwa chakra of Sinchi Warmi Community Tourist Center, located in the parish of Misahuallí port, Napo province. The methodologies implemented to collect the information were ethnography and ethnobotany, which allowed to investigate the historical social interaction of the kichwa Sinchi Warmi community in traditional system of the agricultural productive chakra, from the knowledge dialogue in order to consolidate scientific and traditional knowledge, to develop actions focused on the management and cultivation of plants, taking into consideration the intangible cultural heritage present. As a result, a 60 medical and edible plant species inventory was elaborated. With this information, a guide of food plants and a mobile application for the use of the community, tourists and visitors were created. This exposes a cultural revitalization as an effective alternative to keep alive the ancestral knowledge of this community.

Keywords: ancestral knowledge, cultural and natural heritage, ethnobotany, ethnography, environmental interpretation

\section{RESUMO}

A revitalização cultural tem permitido que saberes, práticas e saberes ancestrais, em risco de desaparecimento, sejam reavaliados e transmitidos às gerações futuras para a gestão sustentável dos seus recursos. Neste contexto, o objetivo da pesquisa foi traçar uma proposta de revitalização do uso de plantas alimentícias no chakra kichwa do Centro de Turismo Comunitário Sinchi Warmi, localizado na freguesia de Puerto Misahuallí, província de Napo. As metodologias implementadas para a recolha de informação foram a etnografia e a etnobotânica, que permitiram indagar sobre a histórica interacção social daquela comunidade no seio do sistema produtivo agrícola tradicional dos chakras a partir do diálogo de saberes, de forma a consolidar conhecimentos científicos e tradicional, e desenvolver ações voltadas ao manejo e cultivo de plantas; tendo em conta o atual patrimônio cultural imaterial. Como resultados, foi obtido um inventário de 60 espécies de plantas com propriedades nutricionais e medicinais; e com isso foram desenvolvidos um guia de plantas alimentícias e um aplicativo móvel para uso das mesmas na comunidade ou por turistas e visitantes. O exposto confirma a revitalização cultural como alternativa efetiva para a salvaguarda dos saberes ancestrais dessas comunidades.

Palavras chave: conhecimento ancestral, etnobotânica, etnografia, interpretação ambiental, patrimônio cultural e natural 


\section{INTRODUCCIÓN}

Uno de los principales problemas que enfrenta hoy la conservación de las prácticas y saberes de las llamadas culturas originarias, es el saber motivar a las generaciones futuras para la salvaguarda de ese patrimonio inmaterial, debido a que la globalización ha impuesto una dinámica socioeconómica que desafía continuamente las costumbres y tradiciones de esos pueblos y nacionalidades.

La revitalización cultural como proceso que posibilita que «aquellas manifestaciones vigentes y que se encuentran en estado de vulnerabilidad puedan ser transmitidas de una generación a otra y sean recreadas constantemente, en tanto otorguen sentido de identidad y pertenencia a una comunidad» (Íñiguez, 2014, p. 5); ha demostrado su potencial para contrarrestar esa realidad, siempre que en ella participen aquellos individuos o grupos portadores de esos conocimientos, prácticas y saberes, con el fin de realizar acciones que generen su sostenibilidad.

El presente estudio tuvo como objetivo diseñar una propuesta para la revitalización del uso de plantas alimenticias en la chakra kichwa del Centro de Turismo Comunitario Sinchi Warmi, localizado en la parroquia Puerto Misahuallí, provincia Napo; pues en ella dicha práctica puede perderse ante el poco interés que muestran las generaciones más jóvenes hacia la aprehensión de esos conocimientos identitarios de su comunidad. Para dar cumplimiento a este propósito, se realizó un marco de referencias que permite el sustento teórico del estudio, un análisis metodológico que facilitó la contextualización de la propuesta a partir de las características de la comunidad y que derivó en herramientas con un marcado carácter interpretativo e ilustrativo de esos saberes, en virtud de contribuir a la salvaguarda de los conocimientos ancestrales.

El Centro de Turismo Comunitario Sinchi Warmi, creado hace aproximadamente 11 años, ha propiciado ya la revitalización del papel de las mujeres kichwas en la producción de alimentos saludables y libres de agroquímicos en las chakras, los que se ofrecen en la actualidad a los turistas o visitantes que llegan a él (Granda, 2017).

Según la Organización de las Naciones Unidas para la Educación, la Ciencia y la Cultura, «el proceso de revitalización sitúa a la comunidad como productora de conocimiento, identidad, valores y características de su herencia y se vuelve tomadora de decisiones respecto a los usos que le pueden dar al conocimiento de su patrimonio» (2003, p. 3).

En este sentido, la chakra kichwa constituye un valioso espacio de confluencias de saberes y prácticas culturales identitarias, al ser un sistema tradicional productivo agrícola caracterizado por el manejo sostenible y biodiverso de sus productos; es decir, que su producción agrícola no solo se maneja de manera orgánica, sino que también les permite a las comunidades amazónicas contribuir a la seguridad alimentaria, al bienestar social de sus miembros y a la conservación de la biodiversidad de la región (Lehmann y Rodríguez, 2013; Torres et al., 2015; Jadán et al., 2015 y Almeida , 2017).

Al respecto, Jadán et al. (2015) afirman que la chakra es considerada como el sistema productivo más importante de la región 
por sus impactos sociocultural, económico y ecológico, los que le permiten adaptarse al cambio climático desde las distintas formas de usanza de la tierra y los elevados niveles de captura de carbono que posee la diversidad de árboles existentes en ella (Torres et al., 2015).

\section{MATERIALES Y MÉTODOS}

En la presente investigación se empleó el enfoque cualitativo para realizar el levantamiento y recolección de la información primaria relacionada con el uso de los saberes ancestrales sobre las plantas en la chakra kichwa, lo que fue de gran importancia para la interpretación y comprensión de estos a través de la percepción y el significado producidos por la usanza de los actores de las plantas existentes en la chakra, desde lo específico a lo general (Hernández et al., 2014).

A través de estudios exploratorios y descriptivos se lograron identificar las características de la comunidad Sinchi Warmi y su estrecha relación con la chakra kichwa y sus productos, con la finalidad de comprender y explorar la problemática relacionada con la pérdida de saberes ancestrales, y generar así, la posibilidad de consumar distintas estrategias que aporten a la salvaguarda y la revitalización del patrimonio cultural inmaterial enfocado al uso de plantas de tipo alimenticio.

Se aplicó el método etnobotánico, que tiene sus antecedentes en la prehistoria del hombre, en el instante en que tomó conciencia de la gran importancia de las plantas para su subsistir (Levy y Aguirre, 1999); para el levantamiento de la información en la fase de campo, donde a su vez se emplearon los métodos propuestos por Kvist et al. (2001) en su artículo Estudio de plantas medicinales en la amazonía peruana: una evaluación de ocho métodos etnobotánicos. Estos métodos se adaptaron a la realidad de la presente investigación, enfocada en las plantas de tipo alimenticio, pero no medicinal; elaborándose una ficha etnobotánica donde se registró todo ese conocimiento.

Figura 1. Métodos de recolección de información.

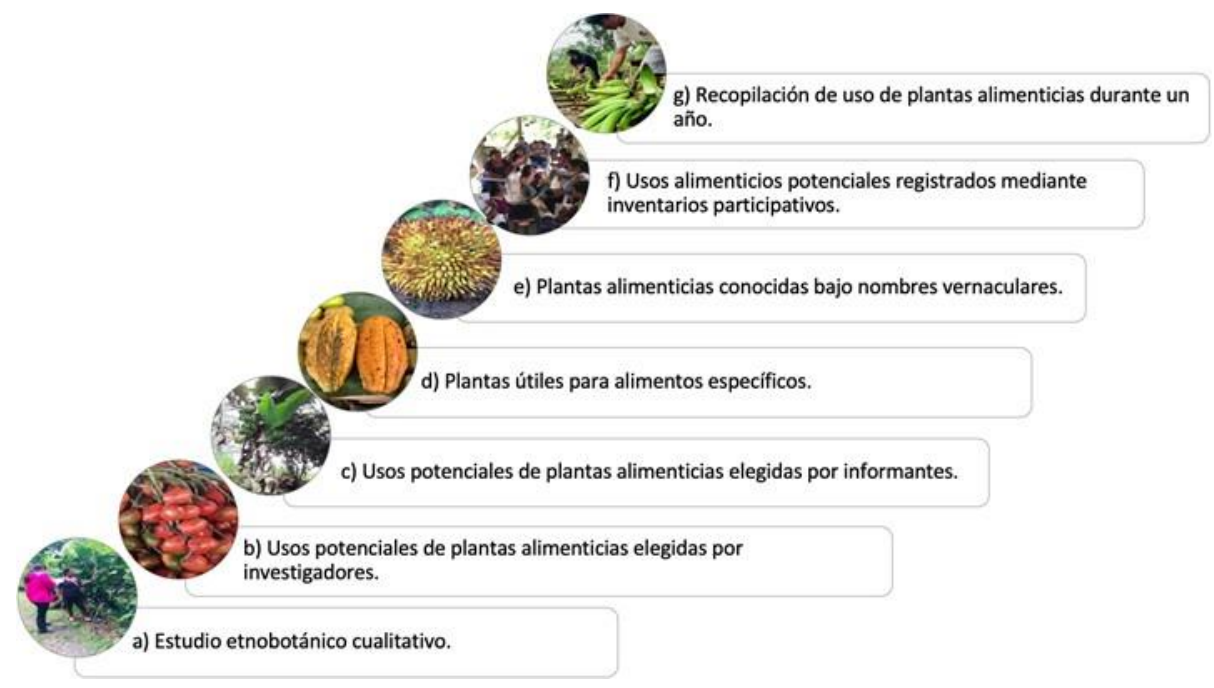

Fuente: Elaboración propia. 
Con la finalidad de comprobar y verificar el uso y registro de los datos obtenidos a través de fuentes secundarias y en la fase de campo, se empleó la técnica del grupo focal (figura 2), al que asistieron 26 personas y donde a través de un diálogo de saberes, se cotejó y verificó el uso de datos como: nombre común, nombre kichwa, hábito, parte empleada, entre otros. Para el registro de la familia y de los nombres científicos de esas especies se realizó una identificación taxonómica en campo (figura 3), además de

Figura 2. Focus group.

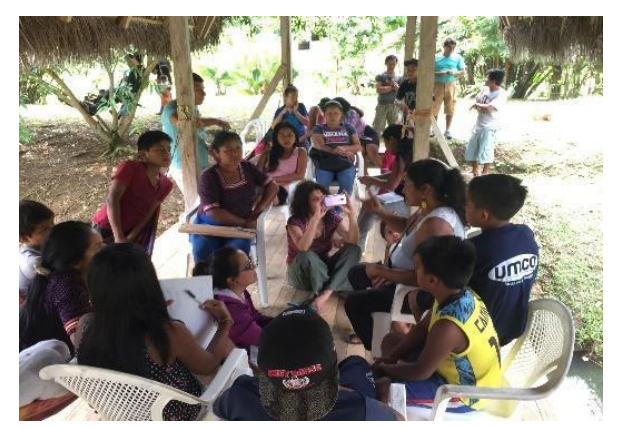

una revisión bibliográfica especializada.

Finalmente se aplicó el método etnográfico para indagar sobre la interacción social histórica de esa comunidad dentro del sistema tradicional productivo agrícola chakra, a partir del diálogo de saberes, a fin de consolidar los conocimientos científicos y tradicionales, y desarrollar acciones enfocadas al manejo y cultivo de plantas; teniendo en cuenta al patrimonio cultural inmaterial presente (Bernal, 2010).

Figura 3. Ficha etnobotánica.

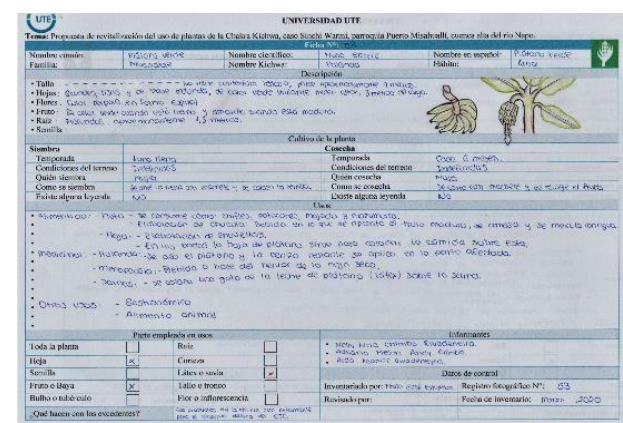

Fuente: Elaboración propia.

\section{RESULTADOS Y DISCUSIÓN}

Como resultados de la investigación se muestra un inventario que registra un total de 60 especies vegetales de tipo alimenticio, presentes dentro de la chakra kichwa del Centro de Turismo Comunitario Sinchi Warmi (anexo 1). El estudio taxonómico de dichas especies expresa la existencia de un total de 35 familias taxonómicas, de las cuales se exponen a continuación seis por ser consideradas las más representativas (figura 4), al contar con un mayor número de especies; el resto de ellas presentó una baja representatividad.

Teniendo en cuenta el hábito de las plantas registradas (figura 5), se evidenció que 28 especies son árboles y 21 son hierbas, mientras que existen cinco especies de arbustos y palmas, respectivamente, y una especie de liana. Estos valores ratifican a los árboles como el hábito más numeroso dentro la chakra kichwa. 
Figura 4. Familias taxonómicas más representativas por mayor número de especies.

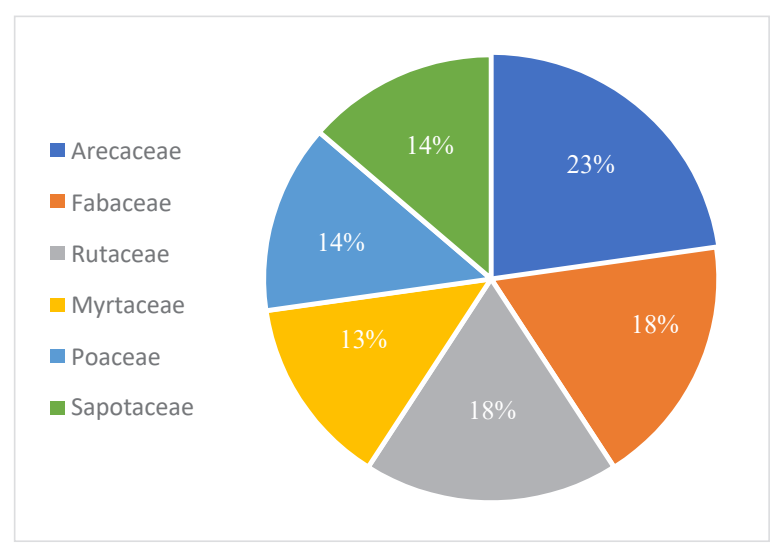

Fuente: Elaboración propia.

Figura 5. Hábito por número de especies presentes en la chakra.

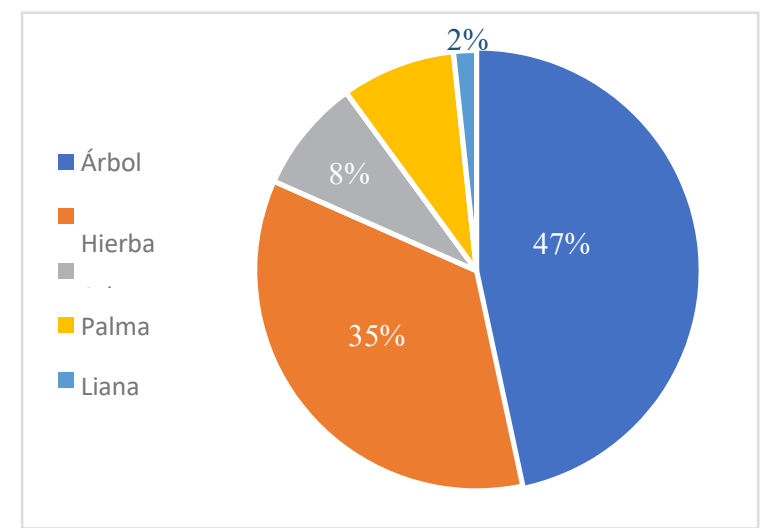

Fuente: Elaboración propia.

Por otra parte, mediante la aplicación de los métodos seleccionados se pudo comprobar que las 60 plantas identificadas son de uso alimenticio para el ser humano, lo cual representa el $100 \%$ del inventario registrado (figura 6). No obstante ello, puede decirse que las mismas cumplen diversas funciones dentro de la chakra kichwa, tales como garantizar el alimento animal: 34 especies, que representan el $29 \%$; diversificar la oferta gastronómica: 32 especies, que representan $27 \%$; con fines terapéuticos medicinales: 31 especies, que representan el $26 \%$; como materia prima para la actividad artesanal: 10 especies, que representan el 9\%; consideradas maderables: 5 especies, que representan el $4 \%$; con valores ornamentales: 4 especies, que representan el $3 \%$; y como fungicida: una sola especie, que representa el $1 \%$. 
Figura 6. Uso de plantas dentro de la chakra kichwa del Centro de Turismo Comunitario Sinchi Warmi.

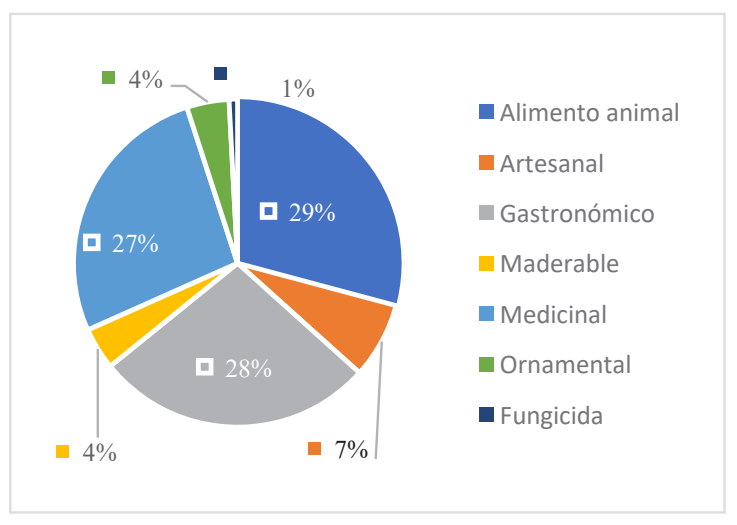

Fuente: Elaboración propia.

Luego de identificar aquellas que poseen un uso alimenticio exclusivo, se procedió a establecer qué parte de la planta es la empleada. Para realizar este análisis se consideraron las siguientes categorías: hoja, cogollo, fruto, raíz, palmito, semilla, corteza, tallo o rizoma, tubérculo; los resultados se expresan en la figura 7 .

Figura 7. Número de especies por parte empleada de la planta.

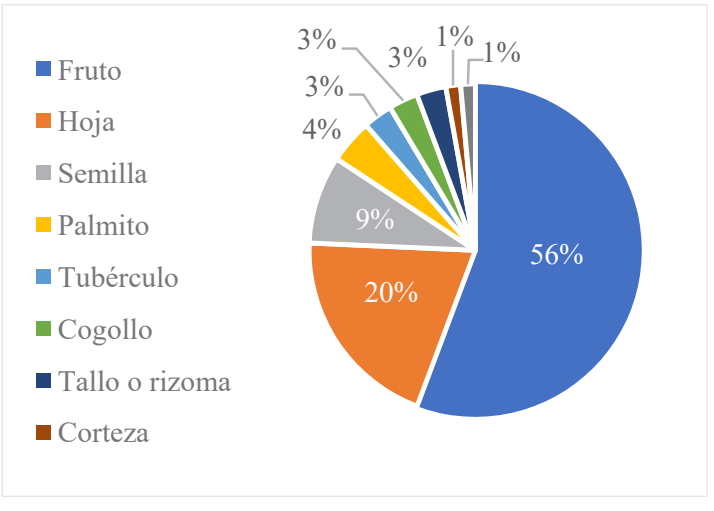

Fuente: Elaboración propia.

Propuesta de revitalización del uso de saberes ancestrales de plantas alimenticias de la chakra kichwa del Centro de Turismo Comunitario Sinchi Warmi

Luego del trabajo de campo en el Centro de Turismo Comunitario Sinchi Warmi, se evidenció la necesidad de tener un registro de información relacionado con el conocimiento del uso de las plantas alimenticias que se encuentran en la chakra kichwa; por lo cual se propuso la elaboración de dos herramientas que permitan revitalizar el empleo de estos saberes ancestrales, con el fin de que varias generaciones actuales y futuras tengan acceso a dicho caudal, considerada patrimonio inmaterial de esa localidad. 
Guía de plantas alimenticias de la chakra kichwa Sinchi Warmi

Esta guía se elabora con el objetivo de socializar las propiedades y empleos de las plantas alimenticias presentes en la chakra kichwa del Centro de Turismo Comunitario Sinchi Warmi, a partir de su valor simbólico y utilitario, como un repositorio de los saberes ancestrales que forman parte de la identidad cultural de esa comunidad. Dentro de dicha guía se encuentran desarrolladas 31 de las 60 especies de plantas identificadas durante el inventario elaborado en la fase de campo (figuras 8, 9, 10 y 11).
Figura 8. Portada de la guía.

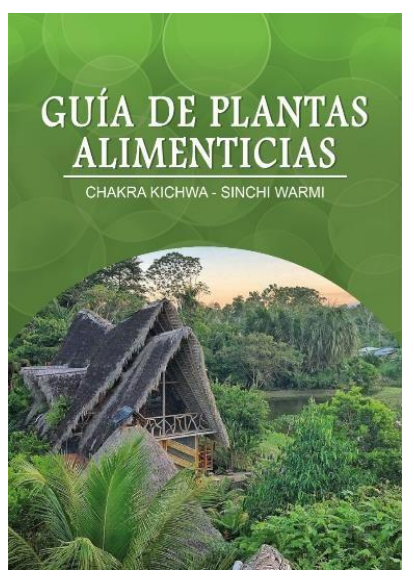

Figura 9. Introducción.

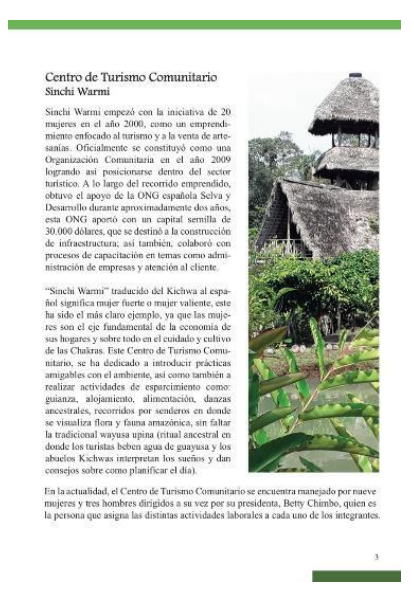

Figura 10. Índice de símbolos.

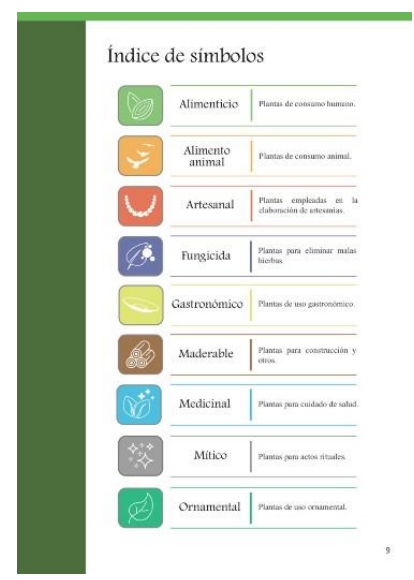

Fuente: Elaboración propia.

Figura 11. Especies desarrolladas.

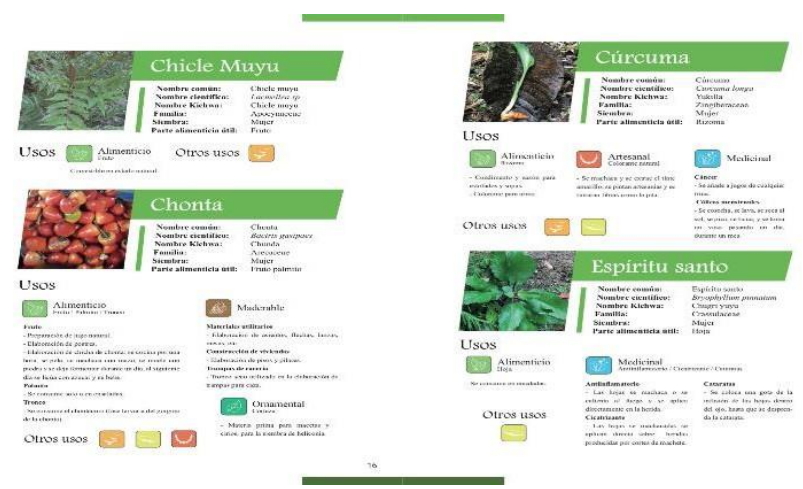

Fuente: Elaboración propia.

\section{Aplicación móvil}

La globalización ha permitido un avance inexorable de la tecnología en todas las sociedades; el Centro de Turismo Comunitario Sinchi Warmi no ha estado exento de ello, por lo cual explotar los beneficios de la misma con el fin de preservar y socializar en un producto informático comunicativo los saberes ancestrales de su comunidad, posibilitó la creación de una aplicación móvil Ilamada Guía de plantas alimenticias 
de la chakra kichwa, que fue elaborada con la información obtenida durante el trabajo de campo (figuras 12 y 13).

La plataforma que se utilizó para la elaboración de este producto fue Mobincube, que de manera gratuita permite crear apps móviles nativas para sistemas operativos como Android y iOS. Esta plataforma genera un alto nivel de personalización con el fin de que la app se adapte a las necesidades requeridas. Asimismo, posibilita su publicación en los principales stores de cada sistema operativo, como son los casos de Google Play y App store. De las 60 especies vegetales obtenidas en el inventario durante la fase de campo, en la aplicación fueron incluidas las 16 especies más utilizadas por sus diversas propiedades dentro de la chakra kichwa.

Figura 12. Diseño de la interfaz gráfica.
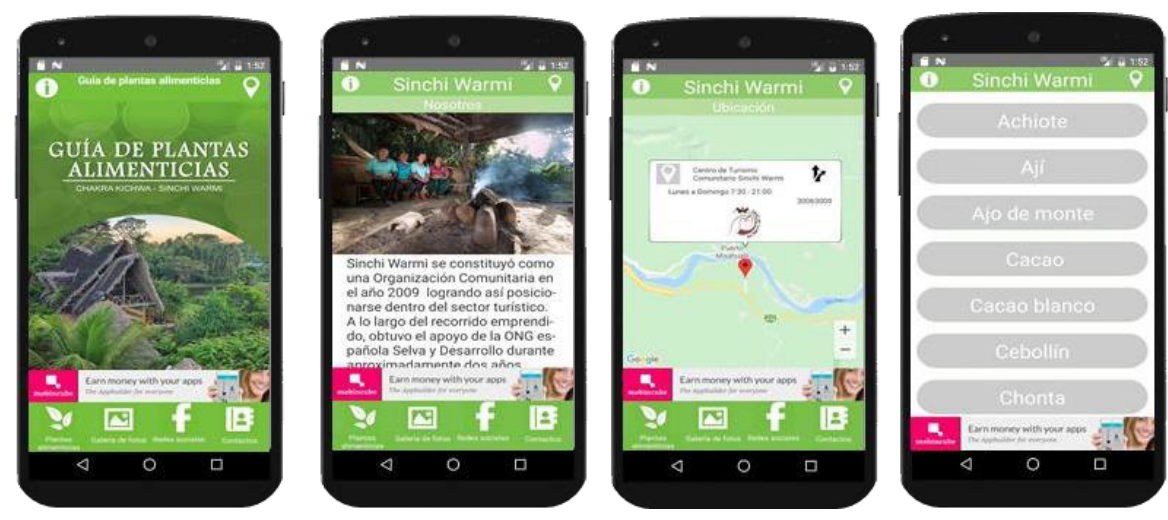

Fuente: Elaboración propia.

Figura 13. Especies desarrolladas.
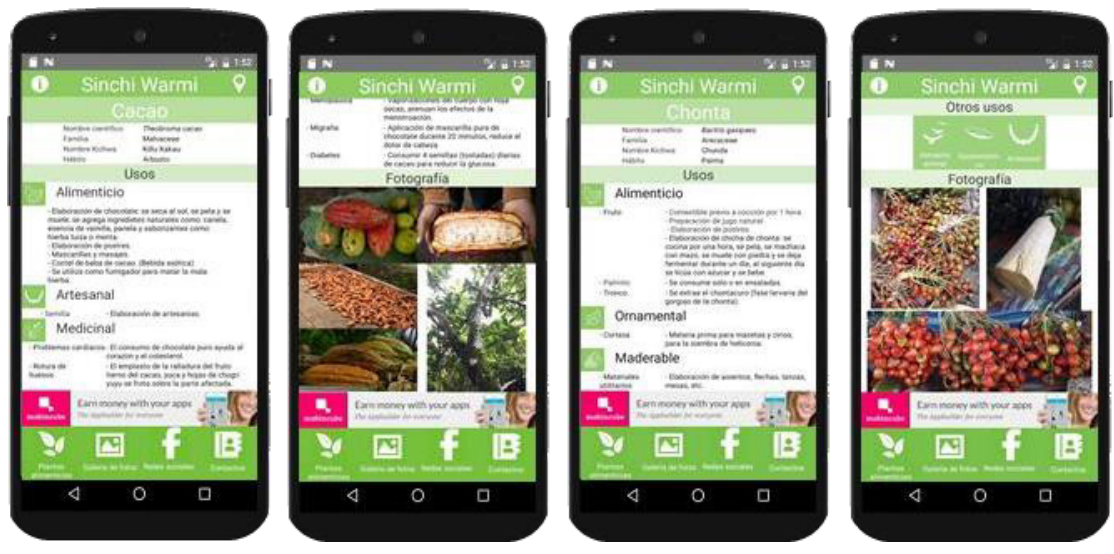

Fuente: Elaboración propia. 


\section{CONCLUSIONES}

La revisión bibliográfica realizada en la presente investigación ratificó el valor simbólico y la importancia socioeconómica que tiene la chakra para las comunidades amazónicas. El Centro de Turismo Comunitario Sinchi Warmi es un fiel exponente de dichos valores no solo por poseer una gran diversidad de especies vegetales que le permiten su adaptación al cambio climático, sino además por su arraigo cultural y la valorización del conocimiento ancestral transmitido por generaciones, basado en el interés de conservar la biodiversidad de la región.

El método etnobotánico permitió utilizar varios parámetros en el levantamiento y registro de 60 especies vegetales de uso alimenticio; y facilitó reconocer a aquellas con propiedades medicinales. Se comprobó que las especies Manihot esculenta (yuca) y Musa ensete (plátano) son las más consumidas y con mayor diversidad de usos por esa comunidad; así como que la chicha que se consume en ella tiene diferentes ingredientes base, tales como: Ipomoea batatas (camote), Zea mays (maíz), Artocarpus altilis (frutipan) y Manihot esculenta (yuca), a diferencia de la elaborada a base de chonta, que constituye una de las bebidas más representativas en la gastronomía de la Amazonía ecuatoriana.

Durante la fase de campo se ratificó la necesidad y el interés del Centro de Turismo Comunitario en registrar de manera interpretativa los distintos usos de las plantas alimenticias en conjunto con sus saberes ancestrales, con el fin de conservarlos y no perderlos a través del tiempo, como ya ha sucedido con otras especies. Cabe mencionar que la presente investigación permitió identificar a las personas claves portadoras de este conocimiento ancestral.

En base a las 31 de las 60 especies de las plantas alimenticias identificadas, se elaboró una guía de plantas alimenticias y una aplicación móvil que permite conocer sus propiedades, cuyo acceso es libre para las personas que se encuentren en el Centro de Turismo Comunitario.

Todo lo expuesto anteriormente ratifica a la revitalización cultural como un proceso necesario y efectivo para la salvaguarda de los saberes ancestrales de estas comunidades.

\section{RECOMENDACIONES}

Realizar investigaciones en las que se amplíe el número de especies, con el fin de generar y alimentar una base de datos consolidada, enfocada al uso y saberes de plantas alimenticias de la chakra kichwa del Centro de Turismo Comunitario Sinchi Warmi.

\section{REFERENCIAS BIBLIOGRÁFICAS}

Almeida, A.E. (2017). La reproducción de la vida: entre la autonomía de la chakra y la dependencia del mercado. Análisis de género en el contexto de la economía social y solidaria en Comunidades Kichwas de Napo [Tesis de maestría, Facultad Latinoamericana de Ciencias Sociales, Ecuador] Repositorio Institucional FLACSO. http://hdl.handle.net/10469/11660 
Bernal, C.A. (2010). Metodología de la investigación: administración, economía, humanidades y ciencias sociales (3ra ed.). Pearson Educación. https://bit.ly/3gi18vu

Granda, E.C. (2017). El Trabajo Social en el fortalecimiento de la práctica ancestral: chacras como medio de identidad y subsistencia familiar de la mujer indígena amazónica en la Asociación Sinchi Warmi de la Comunidad Ancestral Puerto Santa Ana, Pastaza-Puyo. [Tesis de licenciatura, Universidad Central del Ecuador]. Repositorio Institucional UCE. https://bit.ly/3qwaCdi

Hernández, R., Fernández, C., y Baptista, P. (2014). Metodología de la Investigación (6ta ed.). McGraw-Hill. https://bit.ly/3INh3TY

Íñiguez, V. (2014). La revitalización del Patrimonio Cultural Inmaterial. Patrimonio Cultural Inmaterial, 4(13), 5. https://bit.ly/39PMHh9

Jadán, O.G., Günter, S., Torres, B., y Selesi, D. (2015). Riqueza y potencial maderable en sistemas agroforestales tradicionales como alternativa al uso del bosque nativo, Amazonia del Ecuador. Revista Forestal Mesoamericana Kurú, 12(28), 13-22. https://bit.ly/3aHvkFs

Jorgensen, P.M., \& León-Yánez, S. (Eds.) (1999). Catalogue of the vascular plants of Ecuador (Vol. 75). Missouri Botanical Garden Press.

Kvist, L.P., Oré, I., Gonzales, A., y Llapapasca, C. (2001). Estudio de plantas medicinales en la Amazonía Peruana: Una evaluación de ocho métodos etnobotánicos. Folia amazónica, 12(1-2), 53-77. https://bit.ly/39KMxYl

Lehmann, S., y Rodríguez, J. (Coor.) (2013). La Chakra Kichwa: Criterios para la conservación y fomento de un sistema de producción sostenible en la asociación Kallariy sus organizaciones sociales. Serie de sistematizaciones. Fascículo 7. GIZ - Cooperación Alemana. https://bit. $\underline{\text { Iy/3IMyWIM }}$

Levy, T.S., y Aguirre, J.R. (1999). Conceptuación etnobotánica: experiencia de un estudio en la Lacandona. Revista de Geografía Agrícola, 29, 83-114.

Organización de las Naciones Unidas para la Educación, la Ciencia y la Cultura. (2003). Convención para la salvaguardia del patrimonio cultural inmaterial. https://bit.ly/33MjuzX

Royal Botanic Gardens Kew. (2020). Plants of the world on line. http://www.plantsoftheworldonline.org

Torres, B., Maza, O., Aguirre, P., Hinojosa, L., \& Günter, S. (2015). The Contribution of Traditional Agroforestry to Climate Change Adaptation in the Ecuadorian Amazon: The Chakra System. In W. Leal Filho (Eds.), Handbook of Climate Change Adaptation. (pp. 1973-1994). SpringerReference. https://bit.ly/330z0q9 
ANEXO 1

Tabla 1. Inventario de plantas alimenticias de la chakra kichwa del Centro de Turismo Comunitario Sinchi Warmi.

\begin{tabular}{|c|c|c|c|c|}
\hline $\mathbf{N}$ & Nombre común & Nombre Kichwa & Nombre científico & Familia \\
\hline 1 & Achiote & Manduru & Bixa orellana & Bixaceae \\
\hline 2 & Achira & Achira & Canna indica & Cannaceae \\
\hline 3 & Achokcha & Achokcha & Cyclanthera pedata & Cucurbitaceae \\
\hline 4 & Achotillo & Achotillo yura & Nephelium lappaceum & Sapindaceae \\
\hline 5 & Aguacate & Palta & Persea americana & Lauraceae \\
\hline 6 & Ají & Uchu & Capsicum annuum & Solanaceae \\
\hline 7 & Ajo de monte & Sacha aju & Mansoa standleyi & Bignoniaceae \\
\hline 8 & Albahaca & Asnaj panga & Ocimum basilicum & Lamiaceae \\
\hline 9 & Arazá & Araza & Eugenia stipitata & Myrtaceae \\
\hline 10 & Arroz & Arrus & Oryza sativa & Poaceae \\
\hline 11 & Avío & Avio & Pouteria caimito & Sapotaceae \\
\hline 12 & Borojó & Borojo & Alibertia patinoi & Rubiaceae \\
\hline 13 & Cacao & Killu Kakau & Theobroma cacao & Malvaceae \\
\hline 14 & Cacao blanco & Patas muyu & Theobroma bicolor & Malvaceae \\
\hline 15 & Café & Kafia & Coffea arabica & Rubiaceae \\
\hline 16 & Caimito & Kalmitu muyu & Chrysophyllum venezuelanense & Sapotaceae \\
\hline 17 & Camote & Kumal & Ipomoea batatas & Convolvulaceae \\
\hline 18 & Caña de azúcar & Iru & Saccharum officinarum & Poaceae \\
\hline 19 & Cebollín & Suni cebolla & Allium sp. & Amaryllidaceae \\
\hline 20 & Chicle muyu & Chicle muyu & Lacmellea sp. & Apocynaceae \\
\hline 21 & Chirimoya & Ananas & Annona cherimola & Annonaceae \\
\hline 22 & Chonta & Chunda & Bactris gasipaes & Arecaceae \\
\hline 23 & Coco & Coco muyu & Cocos nucifera & Arecaceae \\
\hline 24 & Culantro & Sacha Kulantru & Coriandrum sativum & Apiaceae \\
\hline 25 & Cúrcuma & Yukilla & Curcuma longa & Zingiberaceae \\
\hline 26 & Espíritu santo & Chugri yuyu & Bryophyllum pinnatum & Crassulaceae \\
\hline 27 & Fréjol & Purutu & Phaseolus vulgaris & Fabaceae \\
\hline 28 & Frutipan & Paparu & Artocarpus altilis & Moraceae \\
\hline 29 & Guaba bejuco & Pakai & Inga edulis & Fabaceae \\
\hline 30 & Guaba machetona & Machetona & Inga spectabilis & Fabaceae \\
\hline 31 & Guanábana & Guanabana & Annona muricata & Annonaceae \\
\hline 32 & Guayaba & Guayaba & Psidium guajava & Myrtaceae \\
\hline 33 & Guayusa & Waisa & Ilex guayusa & Aquifoliaceae \\
\hline 34 & Ishpingo & Ishpingu & Ocotea quixos & Lauraceae \\
\hline 35 & Jengibre & Ajiringi & Zingiber officinale & Zingiberaceae \\
\hline 36 & Lima & Llanpun & Citrus sp. & Rutaceae \\
\hline 37 & Limón & Limun & Citrus medica & Rutaceae \\
\hline 38 & Maíz & Sara & Zea mays & Poaceae \\
\hline 39 & Mandarina & Mandarina & Citrus reticulata & Rutaceae \\
\hline 40 & Maní & Inzhij & Arachis hypogaea & Fabaceae \\
\hline 41 & Manzana de agua & Manzana de agua & Syzygium malaccense & Myrtaceae \\
\hline 42 & Morete & Muriti & Mauritia flexuosa & Arecaceae \\
\hline
\end{tabular}

Nota: El nombre común de las plantas fue obtenido en el trabajo de campo con la colaboración de Nelly Chimbo (comunicación personal, marzo, 2020).

Fuente: Los nombres científicos y las familias fueron tomados de Jorgensen \& León-Yánez (1999) y Royal Botanic Gardens Kew (2020). 